\title{
Labyrinthe
}

25 | 2006 (3)

La Bande dessinée : ce qu'elle dit, ce qu'elle montre

\section{La Saveur du monde. Une anthropologie des sens de David Le Breton}

\section{Olivier Wathelet}

\section{OpenEdition}

1 Journals

\section{Édition électronique}

URL : https://journals.openedition.org/labyrinthe/1425

DOI : $10.4000 /$ labyrinthe. 1425

ISSN : 1950-6031

\section{Éditeur}

Hermann

\section{Édition imprimée}

Date de publication : 1 décembre 2006

Pagination : 133-137

ISBN : 2-9526131-2-5

\section{Référence électronique}

Olivier Wathelet, «La Saveur du monde. Une anthropologie des sens de David Le Breton », Labyrinthe [En ligne], 25 | 2006 (3), mis en ligne le 28 mars 2010, consulté le 08 décembre 2022. URL : http:// journals.openedition.org/labyrinthe/1425; DOI : https://doi.org/10.4000/labyrinthe.1425

Ce document a été généré automatiquement le 29 septembre 2020.

Tous droits réservés 


\title{
La Saveur du monde. Une anthropologie des sens de David Le Breton
}

\author{
Olivier Wathelet
}

David Le Breton, professeur de sociologie à l'université March Bloch de Strasbourg, est une référence devenue incontournable dans les débats en sciences humaines portant sur la nature sociale du corps et ce depuis la parution en 1991 de sa fondamentale Anthropologie $d u$ corps et modernité. La thèse essentielle de ce premier ouvragel'effacement ritualisédu corps dans notre société - aura trouvé écho dans une succession d'autres écrits, explorant tantôt les pratiques à risques des adolescents, tantôt les différentes modalités de nos sensibilités mondaines. Avec son dernier essai, La Saveur du monde $e^{*}$, il entend réaliser la synthèse de ses dernières recherches, souligner l'importance de la dimension culturelle dans la structuration sensible du monde et, à plus forte raison, la présence justifiée d'un regard phénoménologique sur le toucher, le goût et les odeurs, dans la boîte à outils méthodologique du chercheur.

Compte tenu de la rareté des travaux s'attachant avec autant d'enthousiasme à dresser les contours systématiques d'une anthropologique des sensations, nous ne pouvons que saluer la parution de ce travail de synthèse. Sur un ton enjoué, l'ouvrage reprend et condense une littérature éparse qui, depuis les travaux du Canadien David Howes au début des années 1990, alimente une réflexion anglo-saxonne pour l'essentiel, et à laquelle l'auteur a personnellement ajouté quelques contributions notables ${ }^{1}$. La Saveur est organisée en neuf chapitres présentant successivement les cinq domaines sensoriels classiques. Ceux-ci proposent ainsi une scansion méthodologique faute de mieux, dont l'auteur lui-même dit regretter l'incapacité à rendre compte avec plus de justesse de la nature synesthésique de notre être au monde et à montrer les liens (de combinaison, association ou opposition) qui composent notre sensibilité. En effet, insiste-t-il, il n'est pas d'engagement singulier qui ne soit de tous les sens, et à plus forte raison, de société humaine qui n'ait développé sa propre culture (multi)sensorielle.

3 Assumant pleinement les spécificités de ce projet d'anthropologie, ces presque cinq cents pages sillonnent le globe et les époques, accumulant anecdotes personnelles, récits de la grande et petite littérature et terrains de patentés ou iconoclastes collègues ${ }^{2}$. Pour 
chaque modalité, avec une multiplication de chapitres consacrés au goût et à la nourriture, « objet sensoriel total » (p. 317), Le Breton souligne la complexité du rapport à soi et à l'autre, les catégories de sens qui émergent des savoirs et savoir-être avec lesquels nous avons appris à faire corps et auxquels nous avons appris à accorder signification. Ce processus, et c'est tout l'intérêt de l'ouvrage que de le souligner, est hautement contextuel, ancré dans des réseaux de comportements et de significations apprises. Entre autres exemples, nous apprenons que chez les Waanzis l'odeur des pêcheurs est considérée comme bonne au moment de leur activité, mais que sitôt de retour au village ils devront s'en débarrasser tant le statut de cette odeur y est alors négatif. Dans le premier cas, cette odeur symbolise la fortune supposée accompagner les pêcheurs. Mais en quittant le contexte précis de leur activité, l'odeur change de valeur et en vient à incommoder (symboliquement et/ou physiologiquement, l'auteur ne le dit pas) ceux qui la perçoivent. De la même manière, et plus proche de nous, on sait, nous rappelle Le Breton, combien les médecins sont capables, le temps d'une consultation, de faire fi du caractère déplaisant des odeurs d'une « mauvaise » hygiène.

4 L'ouvrage consiste ainsi en une somme raisonnée de mises en correspondance d'exemples tirés de différents terrains sensoriels. Un programme ici et là énoncé plus explicitement prend dès lors forme : «L'anthropologie des sens repose sur l'idée que les perceptions sensorielles ne relèvent pas seulement d'une physiologie mais d'abord d'une orientation culturelle laissant une marge à la sensibilité individuelle»(p. 16, nous soulignons).

C'est malheureusement sur ce point théorique que la précision de l'écriture tend à se relâcher. On regrettera tout d'abord, étant donné l'ampleur du projet, la quasi-absence de synthèse transcendant cette accumulation de réflexions et de recensions. Seule cette même ligne de conduite, répétée à maintes reprises, donne le ton : « À chaque instant, à travers son corps, l'individu interprète son environnement et agit sur lui en fonction des orientations intériorisées par l'éducation et l'habitude» (p. 26), orientations que l'agencement des préférences et des goûts individuels - architecture tributaire des aléas singuliers de chaque biographie - nuance et transforme.

6 En des termes aussi généraux, on ne pourrait qu'acquiescer si l'implicite de l'argument, par la réhabilitation d'une figure convenue de la discipline, n'était aussi lourd de conséquences : d'un côté, il y aurait la Culture, de l'autre, la jamais nommée Nature, physiologie des sens et stricte matérialité. Négligeant volontairement la dimension biologique de son propos - réduite à une passive réception d'input - pour affirmer, avec intelligence, le caractère symbolique des techniques/usages du corps sensible, Le Breton s'interdit tout au long de l'écriture de définir clairement les modalités de la médiation entre l'ordre des choses partagées et celui du vécu singulier. Or, à plus forte raison lorsque nous parlons de sensations, expériences singulières s'il en est, il est délicat de manier sans d'infinies précautions (théoriques, méthodologiques) ces deux niveaux d'analyse que sont le collectif (la culture comme ensemble des ressources partagées et d'injonctions intériorisées) et l'individuel (le monde tel qu'ego l'expérimente). Ainsi, en guise d'argument, il suffira à l'auteur de souligner que « la peau est saturée d'inconscient et de culture» (p. 178) ou que "si l'enfant occidental dépend de son ouïe pour son éducation, celle-ci n'est pour l'enfant africain qu'une médiation parmi d'autres » (p. 170), assertions dont le caractère aussi flou que péremptoire ne saurait suffire à l'exigence de précision que l'auteur s'était lui-même imposée. 
7 Aussi, corollaire de ce premier grief, l'insistance avec laquelle est défendu le caractère visuo- et audiocentré des univers occidentalisés, pourtant contrebalancé par la nature même de la démarche de cet ouvrage, à savoir rendre compte du caractère plurisensoriel de notre être au monde, fait partie de ces traits grossiers que nous aurions aimé voir travaillés avec plus de précision. De l'ouvrage se dégage un panorama que nous pouvons difficilement suivre, celui d'un ailleurs, temporel et spatial, équilibré sensuellement, et d'un présent occidental perverti, dont la profusion du bruit, la surcharge visuelle ou l'appauvrissement de la palette des goûts seraient les traits les plus saillants. À notre sens, nous retrouvons ainsi dans ce projet d'analyse des sensations un paradoxe parfois difficilement tenable dans l'œuvre de Le Breton: l'affirmation d'une perte sociale du corps, et son omniprésence dans le propos. Pour rendre encore plus clair ce parallèle, nous pourrions dire que tout en œuvrant à la reconnaissance du caractère plurisensoriel de nos quotidiens, il défend avec vigueur l'hypothèse d'une sensorialité occidentale appauvrie (par rapport à un ailleurs et un avant) dans les domaines du toucher, du goût ou de l'olfaction. Cependant, pour défendre une assertion de ce type, il nous semble avant tout nécessaire de définir précisément ce qu'est une expérience sensorielle et, dans un second temps, quelles sont les propriétés de celle-ci qui puissent subir l'épreuve de la comparaison. Or, ce travail, précisément, est celui qui fait ici le plus cruellement défaut à l'analyse. En raison de ce flottement dans la caractérisation des relations entre l'expérience d'être au monde d'une part et la nature symbolique et rituelle du social d'autre part, on peut regretter que certaines notions, telle celle d'éducation sensorielle, devant assurer la synthèse entre ces deux niveaux d'analyse, ne soit pas clairement définie, empêchant ainsi d'en démontrer les effets. Si tant est que la place du corps dans nos sociétés modernes a été radicalement modifiée par les transformations technologiques et idéologiques récentes, une théorie du social plus nuancée sachant faire la part des choses entre le discours public (celui avec lequel semble travailler l'auteur) et celui de l'intime (affirmé mais rarement mobilisé concrètement et que seul un travail ethnographique en profondeur pourrait révéler) nous permettrait d'avancer avec plus de précision dans la compréhension de ces transformations.

8 En l'absence de cette assise - tant de méthode que de système -, la description des habitudes collectives, des normes et des praxis singulières, aussi érudite soit-elle, justifie plus que de besoin la démarche, mais la contraint simultanément à ne pas en dire beaucoup plus sur nos sensibilités. L'observation d'indigènes experts à la chasse ou le partage d'un même univers odorant suffit-il à assurer une transmission culturelle des manières de sentir (si tant est que nous mettions au centre de notre propos le faire, et non les savoirs, point délicat sur lequel l'auteur se fait également relativement discret) ? Rien ne nous paraît moins évident, à plus forte raison si le grand absent de la réponse est la nature biologique du corps lui-même.

9 Accordons que, fort modestement, l'auteur précise en sous-titre ne proposer qu'« une anthropologie des sens ». Dès lors, nous ne saurions que souligner cette invitation implicite à appliquer ce même enthousiasme à une autre perspective, et souhaiter une anthropologie sensorielle qui, comme le rappelait récemment Jean-Pierre Albert, ne se risquerait pas à faire l'économie d'un dialogue avec les sciences de la cognition ${ }^{3}$. Avec davantage de précision dans la caractérisation de son objet, cette autre anthropologie devrait prendre à bras-le-corps la question des actes singuliers, en posant nécessairement la question de l'existence et de l'organisation des «sentirs partagés", plutôt que de 
s'atteler à décrire ces formes floues, aux contours mal identifiés que sont des «cultures sensibles » aux corps effacés.

\section{NOTES}

*. Paris, Métailié, coll. « Traversées », 2006, 452 p., $20 €$.

1. Parmi les références œuvrant plus explicitement à une anthropologie des sens, on retiendra Les Passions ordinaires, Paris, Armand Colin, 1998; La Chair à vif, Paris, Métailié, 1999 ; Du silence, Paris, Métailié, 2001 ; La Peau et la Trace, Paris, Métailié, 2003... et, de manière plus significative, sa codirection avec Colette Méchin et Isabelle Bianquis-Gasser de Anthropologie du sensoriel, Paris, L'Harmattan, 1998. On retiendra en outre que ce volume est le premier à employer explicitement, dans l'univers francophone européen, la notion d'anthropologie du sensoriel.

2. La liste est particulièrement longue. Outre les classiques : Bachelard, Elias, Foucault, Freud et Lévi-Strauss, on retrouve quelques figures marquantes des recherches contemporaines en matière de sensibilité historique et sociale: Pierre Camporesi, Joël Candau, Constance Classen, Alain Corbin, Jean-Louis Flandrin, Edward T. Hall, David Howes, Marshal Mac Luhan, Walter Ong, Michel Serres ou Paul Stoller pour ne citer que les plus significatifs. On sera agréablement surpris de découvrir, à côté de ce répertoire presque obligé, une longue liste de travaux moins connus dans le champ, mais dont l'intérêt ne manque pas.

3. Jean-Pierre Albert, « Parfum \& mysticisme », dans Voir, 28-29, (novembre) 2004, p. 42-56.

\section{AUTEUR}

\section{OLIVIER WATHELET}

Owathelet[at]gmail.com 